\title{
Activation of Tyrosine Metabolism in CD13+ Cancer Stem Cells Drives Relapse in Hepatocellular Carcinoma
}

Li Sun, $M D, P h D^{1}$
Lin Zhang, MD²
Jun Chen, $\mathrm{MD}^{3}$
Chaoqun Li, MD
Hongqin Sun, MD
Jiangrong Wang, MD
Hong Xiao, MD

${ }^{1}$ Department of Oncology, Renji Hospital, School of Medicine, Shanghai Jiaotong University, Shanghai, ${ }^{2}$ The Nursing Department, ${ }^{3}$ The First Department of Infectious Diseases, ${ }^{4}$ The Third Department of Infectious Diseases, Shanghai Public Health Clinical Center, Shanghai, China
Correspondence: Hong Xiao, MD

The Third Department of Infectious Diseases,

Shanghai Public Health Clinical Center,

Shanghai 201508, China

Tel: 86-21-3799-0333

Fax: 86-21-3799-0333

E-mail: xiaohong20190709@hotmail.com

Received August 6, 2019

Accepted December 26, 2019

Published Online December 27, 2019

\begin{abstract}
Purpose
Cancer stem cells (CSCs) are naturally resistant to chemotherapy, explaining why tumor relapse frequently occurs after initial regression upon administration of chemotherapeutic agents in most cases. A CSC population characterized by CD13 expression has been identified in hepatocellular carcinoma (HCC). In the current study, we aimed to clarify the molecular mechanism by which it escapes conventional therapies.
\end{abstract}

\section{Materials and Methods}

Here, we used flow cytometry to examine the percentage of CD13+ CSCs in HepG2 and $\mathrm{HuH7}$ cells after chemotherapy. Using in vitro isotope labeling technique, we compared metabolic pathways between $\mathrm{CD}_{13}{ }^{+}$and $\mathrm{CD} 13^{-}$subpopulations. Using co-immunoprecipitation and western blotting, we determined the target expressions in protein levels under different conditions. We also performed immunohistochemistry to detect the target proteins under different conditions. Animal models were constructed to verify the potential role of tyrosine metabolism in post-chemotherapeutic relapse in vivo.

\section{Results}

We observed that quiescent $\mathrm{CD} 13^{+} \mathrm{CSCs}$ are enriched after chemotherapy in HCCs, and serve as a reservoir for recurrence. Mechanistically, $\mathrm{CD} 13^{+} \mathrm{CSCs}$ were dependent on aerobic metabolism of tyrosine rather than glucose as energy source. Tyrosine metabolism also generated nuclear acetyl-CoA to acetylate and stabilize Foxd3, thereby allowing $\mathrm{CD} 13^{+} \mathrm{CSCs}$ cells to sustain quiescence and resistance to chemotherapeutic agents.

\section{Conclusion}

These findings encourage further exploration of eliminating $\mathrm{CD} 13^{+}$cells by targeting specific metabolic pathways to prevent recurrence in HCCs.

\section{Introduction}

Hepatocellular carcinoma (HCC) is the fifth most commonly diagnosed cancer and the second most frequent cause of cancer death in men worldwide [1]. The treatment algorithms in the American Association for the Study of Liver Disease (AASLD) and the European Association for the Study of the Liver (EASL) and the European Organization for Research and Treatment of Cancer (EORTC) guidelines
Key words

CD13 ${ }^{+}$CSCs, Foxd3, Hepatocellular carcinoma, Quiescence, Tyrosine metabolism recommend resection and ablation or transplantation for early-stage HCC, transcatheter arterial chemoembolization for intermediate-stage HCC and sorafenib (SR) for advancedstage HCC [2,3]. Unfortunately, cancer cells often develop chemoresistance, which presents a major obstacle to the long-term efficacy of chemotherapeutic treatments. There is increasing evidence that resistance to chemotherapy is, at least in part, caused by inherent resistance of a subpopulation of cancer cells, which have been labeled as cancer stem cells (CSCs) [4]. Liver CSCs are considered to be responsible 
for tumor growth, metastasis and recurrence of HCC as well as for the failure of chemotherapy and radiotherapy [5]. The traditional strategies leave behind residual CSCs which possess the abilities of self-renewal, multi-directional differentiation, and indefinite proliferation, as well as high tumorigenic ability [4], leading to cancer recurrence and metastasis. Therefore, the cancer stem cell theory offers novel insight into tumor diagnosis, treatment, and prevention.

Several markers have been proposed in the literature to identify CSCs in HCCs using cell surface antigens [6,7]. Recently, Haraguchi et al. [8] identified CD13, a membranebound zinc-dependent type II exopeptidase, as a candidate liver cancer stem cell marker by a surface marker screen based on microarray analysis. CD13 ${ }^{+}$CSCs were enriched in side population, which is known as the multidrug-resistant cell fraction with ATP-binding cassette transporter expression, and also localized predominantly in G1/G0 phase [8,9]. Thus, $\mathrm{CD}^{+}{ }^{+}$cells represent the dormant or slow-growing population that is believed to account for the chemoresistant capacity in HCC. In this study, we observed selective enrichment of $\mathrm{CD} 3^{+}$cells following chemotherapies in HCC, suggesting that $\mathrm{CD}_{13}{ }^{+} \mathrm{CSC}$ s are major reservoir for eventual recurrence for HCCs. Suppressing residual CD13 ${ }^{+}$CSCs after initial tumor debulking may sustain remissions and extend the progression-free survival of patients receiving CSC suppressive therapy. However, this task is complicated by the fact that CD13 is widely distributed in many tissues of mammals, such as the intestine, kidney, and liver as well as the central nervous system [10]. Until these challenges are overcome, CSC-targeting therapies will not reach their full potential.

Emerging evidence suggests metabolic reprogramming as a key player in CSC biology [11]. The action of metabolic pathways in CSC maintenance might not be merely the consequences of genomic alterations. Indeed, the metabotypic phenotypes may play a causative role in maintaining the stem traits. Therefore, targeting the metabolic events represents a potential strategy to halt tumor recurrence and refractoriness to treatment $[12,13]$. As opposed to differentiated bulk tumor cells which can be highly glycolytic or oxidative phosphorylation dependent, it is difficult to univocally identify the metabolic phenotypes that maintain CSC stemness. Due to the different microenvironmental conditions they survive in, CSCs are most likely supposed to get energy from different sources, according to substrate availability [11]. Based on the facts above, we further conducted a series of experiments to examine the metabolic phenotypes that render CD13+ CSCs multidrug-resistant in HCCs.

\section{Materials and Methods}

\section{HCC cell lines}

Huh7 and HepG2 cells were obtained from American Type Culture Collection and cultured in RPMI-1640 medium (Invitrogen, Carlsbad, CA) supplemented with 10\% fetal bovine serum (Invitrogen).

\section{Flow cytometry and cell sorting}

Cells were incubated in phosphate-buffered saline containing $2 \%$ fetal bovine serum with phycoerythrin-conjugated anti-human CD13 antibody (Becton Dickinson, San Jose, CA). Isotype-matched mouse immunoglobulins served as controls. For flow cytometry, samples were analyzed using a FACSCalibur flow cytometer and CellQuest software (BD Biosciences, San Jose, CA). For cell sorting by flow cytometry, samples were analyzed and sorted on a BD FACSVantage SE (BD Biosciences). For the positive and negative population, only the top $25 \%$ most brightly stained cells or the bottom $20 \%$ most dimly stained cells were selected, respectively. Aliquots of $\mathrm{CD}^{3} 3^{+}$and $\mathrm{CD}^{2} 3^{-}$sorted cells were evaluated for purity with a FACSCalibur machine and CellQuest software (BD Biosciences), using PE-conjugated anti-human CD13 antibody.

\section{In vitro isotope labeling and kinetic profiling}

To trace glucose, glutamine or tyrosine metabolism, $\mathrm{CD} 13^{+}$ cells were grown as tumorspheres in growth media (Dulbecco's modified Eagle's medium/F12 nutrient mixture Ham [DMEM/F12]) on $10 \mathrm{~cm}$ dishes and then transferred into glucose-, glutamine- or Tyr-free DMEM/F12 medium supplemented with ${ }^{13} \mathrm{C}$-glucose, ${ }^{13} \mathrm{C}$-glutamine, and ${ }^{13} \mathrm{C}$-tyrosine (Cambridge Isotope Labs, Tewksbury, MA) to $10 \mathrm{mM}$ (for glucose), $2 \mathrm{mM}$ (for glutamine), or $0.1 \mathrm{mM}$ (for Tyr) overnight (for steady-state labeling) or for the indicated time points in the flux analyses. Additionally, fresh media containing ${ }^{13} \mathrm{C}$-glucose, ${ }^{13} \mathrm{C}$-glutamine, and ${ }^{13} \mathrm{C}$-tyrosine was exchanged 2 hours prior to metabolite extraction for steadystate analyses.

\section{Statistical analysis}

All statistical analyses were carried out with SPSS ver. 22 (IBM Corp., Armonk, NY). ANOVA, Student's t test, and Dunnett's multiple comparison tests were used to compare mean values. Data are presented as mean \pm standard deviation. A p-value of $<0.05$ was defined as statistically significant. Other methods are shown in Supplementary Methods. 


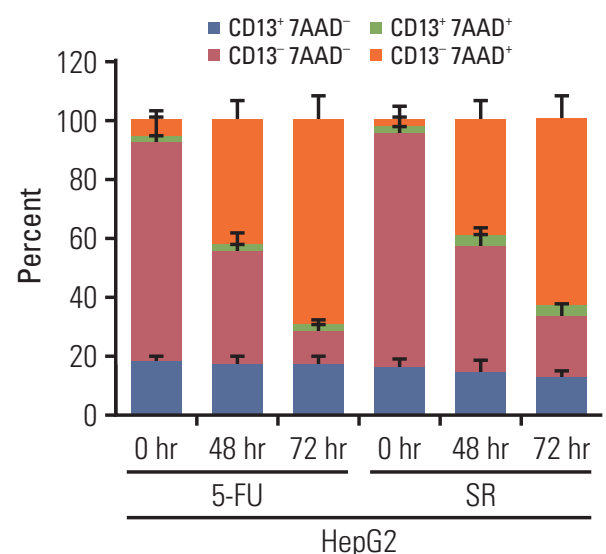

B

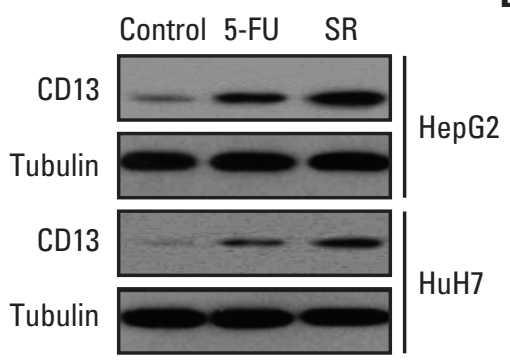

D

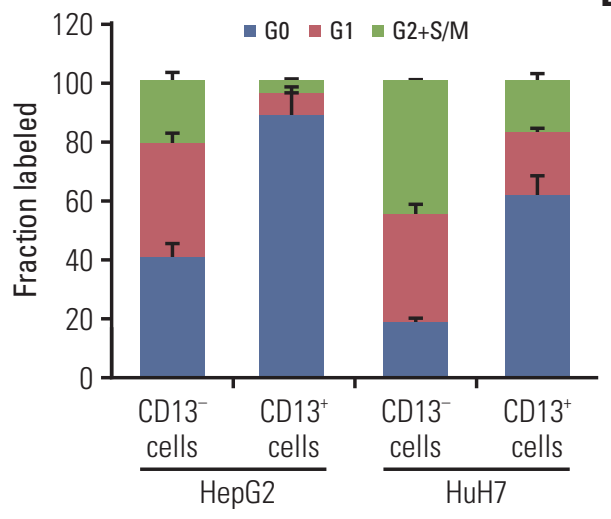

A

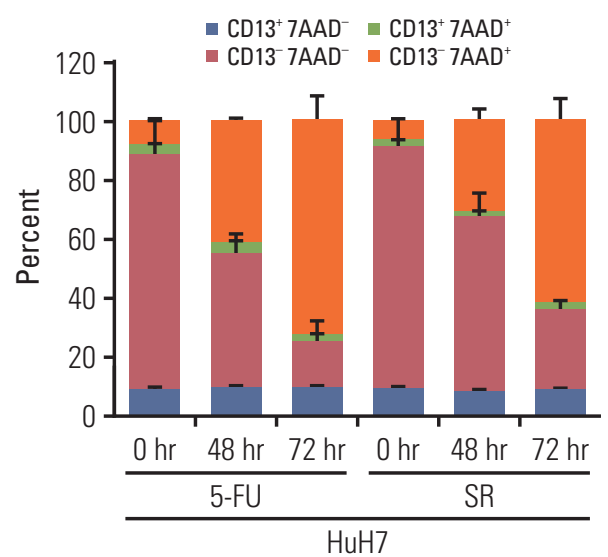

C

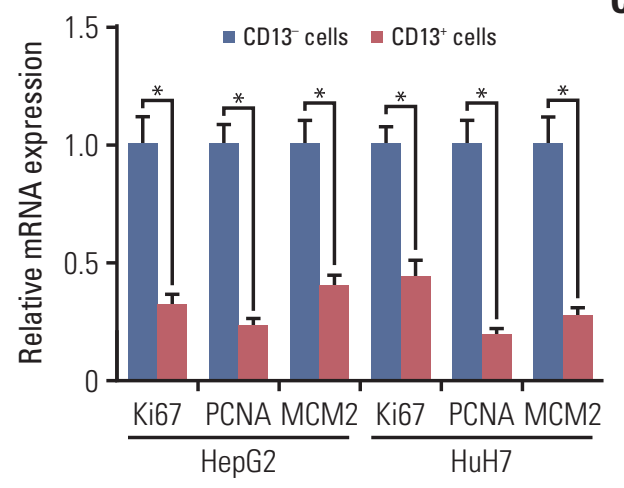

E

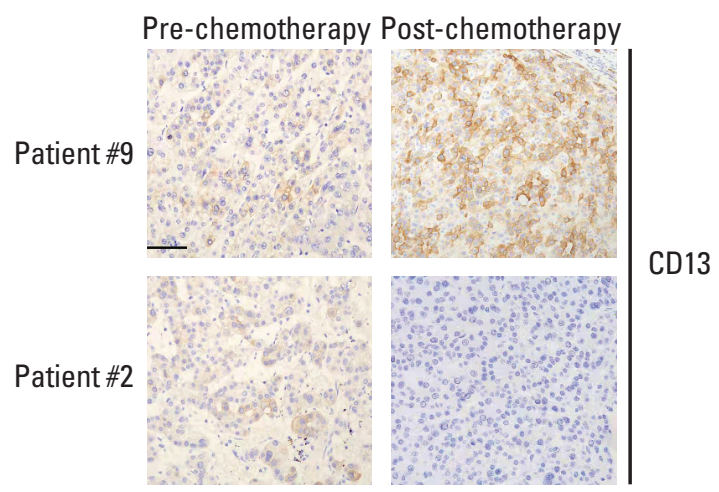

Fig. 1. Quiescent $\mathrm{CD}_{13}{ }^{+}$cancer stem cells in hepatocellular carcinomas (HCCs) are enriched following chemotherapy. (A) HepG2 and HuH7 cells were persistently treated with 5-fluorouracil (5-FU; $5 \mu \mathrm{M})$ or sorafenib (SR; $4 \mu \mathrm{M})$ and analyzed for CD13 expression by flow cytometry at the indicated time points. Dead cells were detected by 7AAD staining. (B) Immunoblotting of CD13 protein expression in pre- and drug-treated HepG2 and HuH7 cells at 48-hour post-treatment. (C) Representative markers for proliferation were examined at the mRNA level in CD13 ${ }^{+}$and $\mathrm{CD}_{13}^{-}$subsets from HepG2 and HhH7 cells. (D) Cell cycle distribution of $\mathrm{CD}_{13}^{+}$and $\mathrm{CD} 13^{-}$fractions from HepG2 or HuH7 cells was determined by combined staining with Hoechst33342 and pyroninY. (D) Cell cycle distribution of CD13 ${ }^{+}$and CD13- fractions from HepG2 or HuH7 cells was determined by combined staining with Hoechst33342 and pyroninY. (E) Representative images of $\mathrm{CD}_{13}{ }^{+}$cell frequency in untreated tumors and tumor remnants after hepatic arterial infusion chemotherapy evaluated by immunohistochemistry (IHC). Scale bar $=50 \mu \mathrm{m}$. (Continued to the next page) 

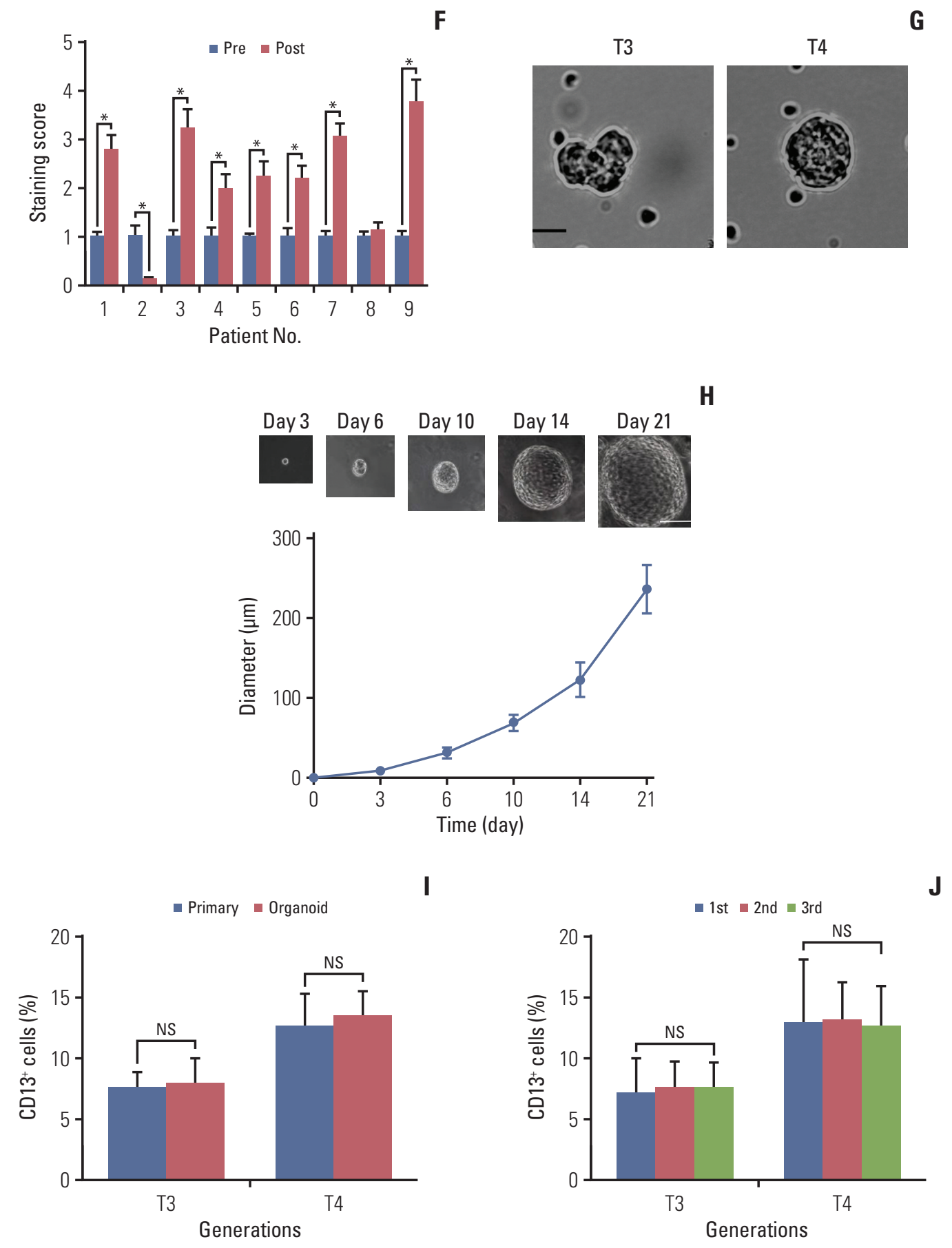

Fig. 1. (Continued from the previous page) (F) Comparison of IHC CD13 staining from nine HCC patients before and after hepatic arterial infusion chemotherapy. All patients achieved a partial regression. (G) Representative images of primary 3D organoids on day 12 from tumor biopsies of two HCC patients (T3 and T4). Scale bar=100 $\mu \mathrm{m}$. (H) The representative images of T4-derived 3D organoids are shown (upper) along with the corresponding growth curve (bottom). Scale bar=150 $\mu \mathrm{m}$. (I) Quantification analysis of $\mathrm{CD}_{13}{ }^{+}$cells in primary $\mathrm{T} 3 / \mathrm{T} 4$ tumors and their corresponding organoids. (J) Flow cytometric analysis of $\mathrm{CD}_{13}^{+}$cells in $\mathrm{T} 3$ and T4-derived organoids during serial passages. (Continued to the next page) 
T3

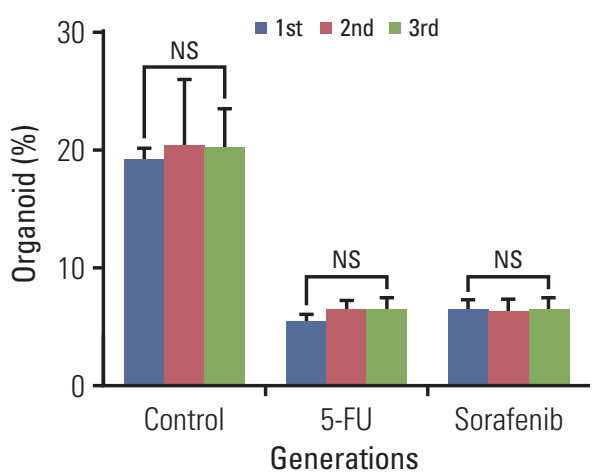

T3

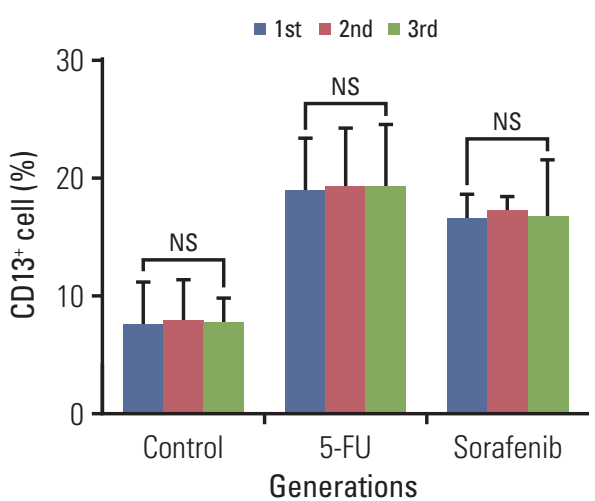

T4

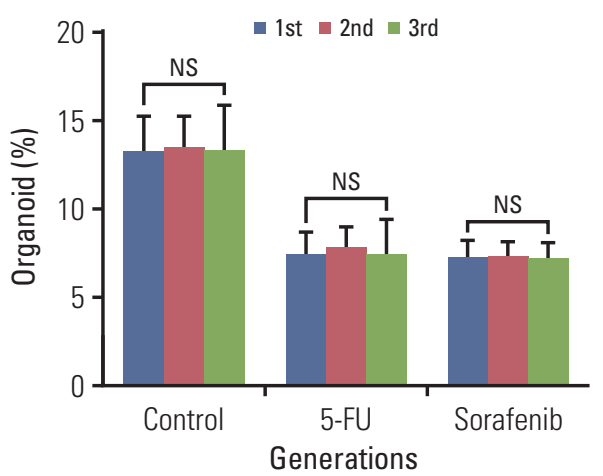

T4

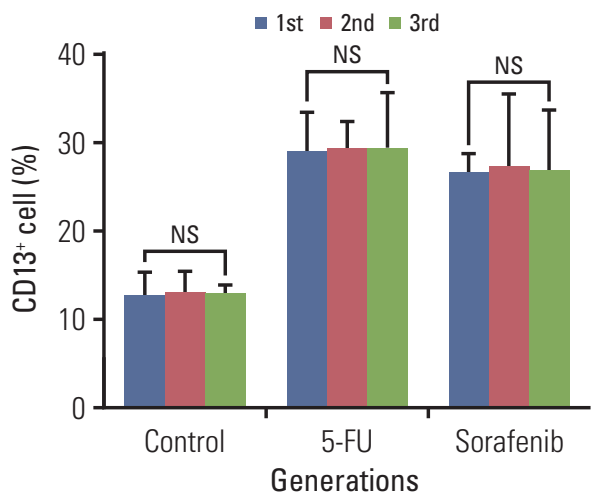

Fig. 1. (Continued from the previous page) (K) Organoid-forming efficiency in the serial organoid cultures upon the administration of chemotherapeutic agents ( $5 \mu \mathrm{M} 5$-FU or $4 \mu \mathrm{M}$ sorafenib). (L) Flow cytometric quantification analysis of CD13 ${ }^{+}$cells in the serial organoid cultures upon administration of chemotherapeutic agents ( $5 \mu \mathrm{M} 5$-FU or $4 \mu \mathrm{M}$ SR). Values shown are mean \pm standard deviation. A two-tailed unpaired t test was used to compare experimental groups. ${ }^{*} \mathrm{p}<0.05$; NS, no significance.

\section{Ethical statement}

Human HCC specimens were obtained with written informed consent from all subjects before surgery and in accordance with the ethical standards of the Institutional Review Board of HuaShan Hospital (No. 20170036). The collection and processing of all samples were carried out in accordance with the Declaration of Helsinki. All mouse experiments were conducted in accordance with standard operating procedures approved by the Institutional Animal Care and Use Committee for Shanghai Public Health Clinical Center (protocol No. 2018X112) and adhered to the Animals in Research: Reporting In Vivo Experiments (ARRIVE) Guidelines.

\section{Results}

\section{Selective enrichment of quiescent $\mathrm{CD}^{+}{ }^{+} \mathrm{CSC}$ following chemotherapy in HCCs}

Analysis of HepG2 cells by flow cytometry showed that $\mathrm{CD}^{-}{ }^{-}$cells were a majority $(82.3 \% \pm 6.9 \%)$ of the total population before treatment. However, the viable CD13- population was reduced to $15.3 \% \pm 3.1 \%$ after exposure to 5 -fluorouracil (5-FU; $5 \mu \mathrm{M}$ ) for 72 hours, while the viable CD13 ${ }^{+}$ population increased from $8.4 \% \pm 2.3 \%$ to $72.1 \% \pm 5.2 \%$ (Fig. $1 \mathrm{~A}$ left). Similar results were observed in HepG2 cells after exposure to SR $(4 \mu \mathrm{M})$ (Fig. 1A left). Immunoblotting further confirmed a significant enrichment for endogenous CD13 protein (Fig. 1B). Similarly, neither 5-FU nor SR failed to eradicate all HCC cells and enriched for $\mathrm{CD}_{13}{ }^{+}$cells in $\mathrm{HuH7}$ 


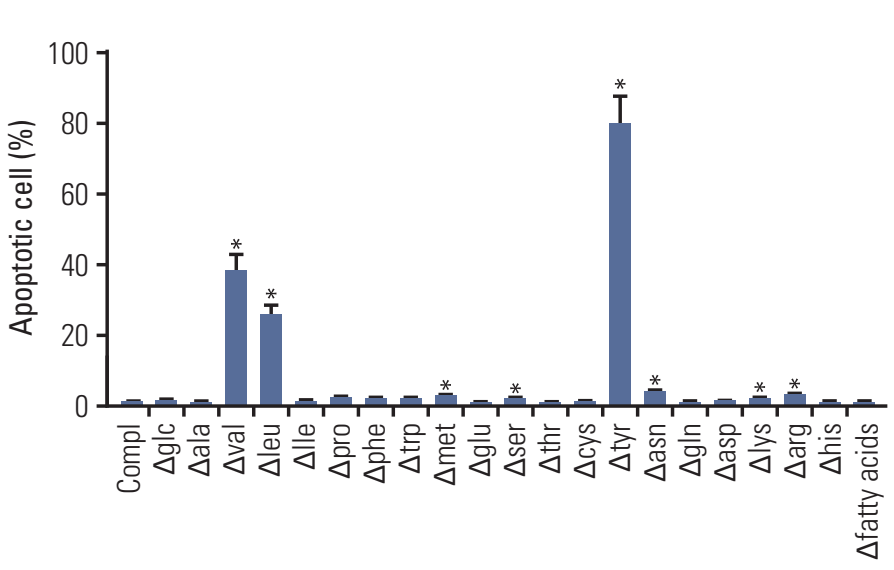

A

B
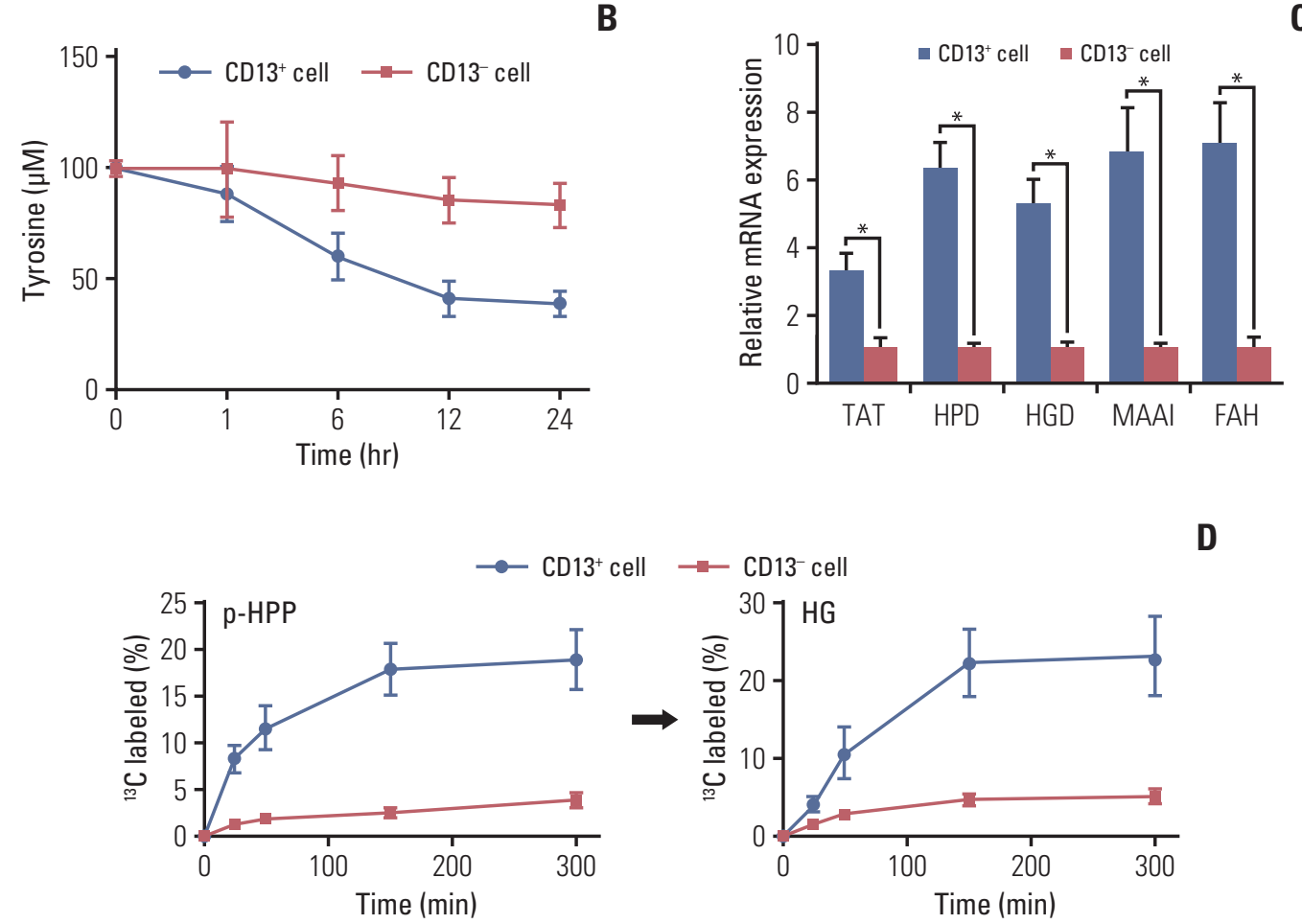

D
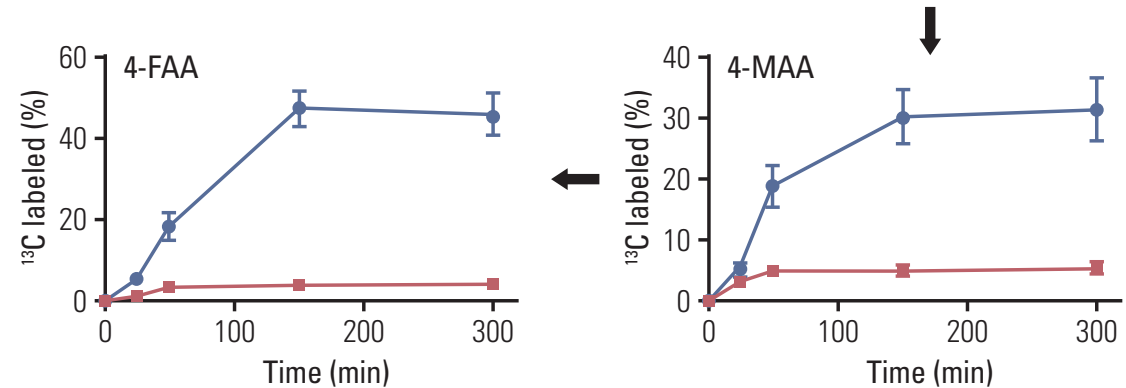

Fig. 2. Tyr metabolism is enhanced in $\mathrm{CD} 13^{+}$cancer stem cells from hepatocellular carcinomas. (A) Apoptosis in HepG2derived $\mathrm{CD} 13^{+}$cells was determined 72 hours after deprivation of essential nutrients. (B) Comparison of Tyr consumption in HepG2-derived CD13- vs. CD13 ${ }^{+}$cells. (C) Comparison of mRNA level of enzymes in Tyr metabolism in HuH7-derived $\mathrm{CD}^{-}{ }^{-}$vs. CD13 ${ }^{+}$cells. (D) HepG2-xenografted BALB / c nude mice received ${ }^{13} \mathrm{C}$-tyrosine infusion prior to cytometric sorting of $\mathrm{CD}_{13}{ }^{+}$and $\mathrm{CD} 13^{-}$cells. The percentage of ${ }^{13} \mathrm{C}$-labeled intermediates (para-hydroxyphenylpyruvate[p-HPP], homogentisic acid [HG], 4-maleylacetoacetate [4-MAA], and 4-fumarylacetoacetate [4-FAA]) was assessed by mass spectrometry ( $\mathrm{n}=3$ per time point). (Continued to the next page) 

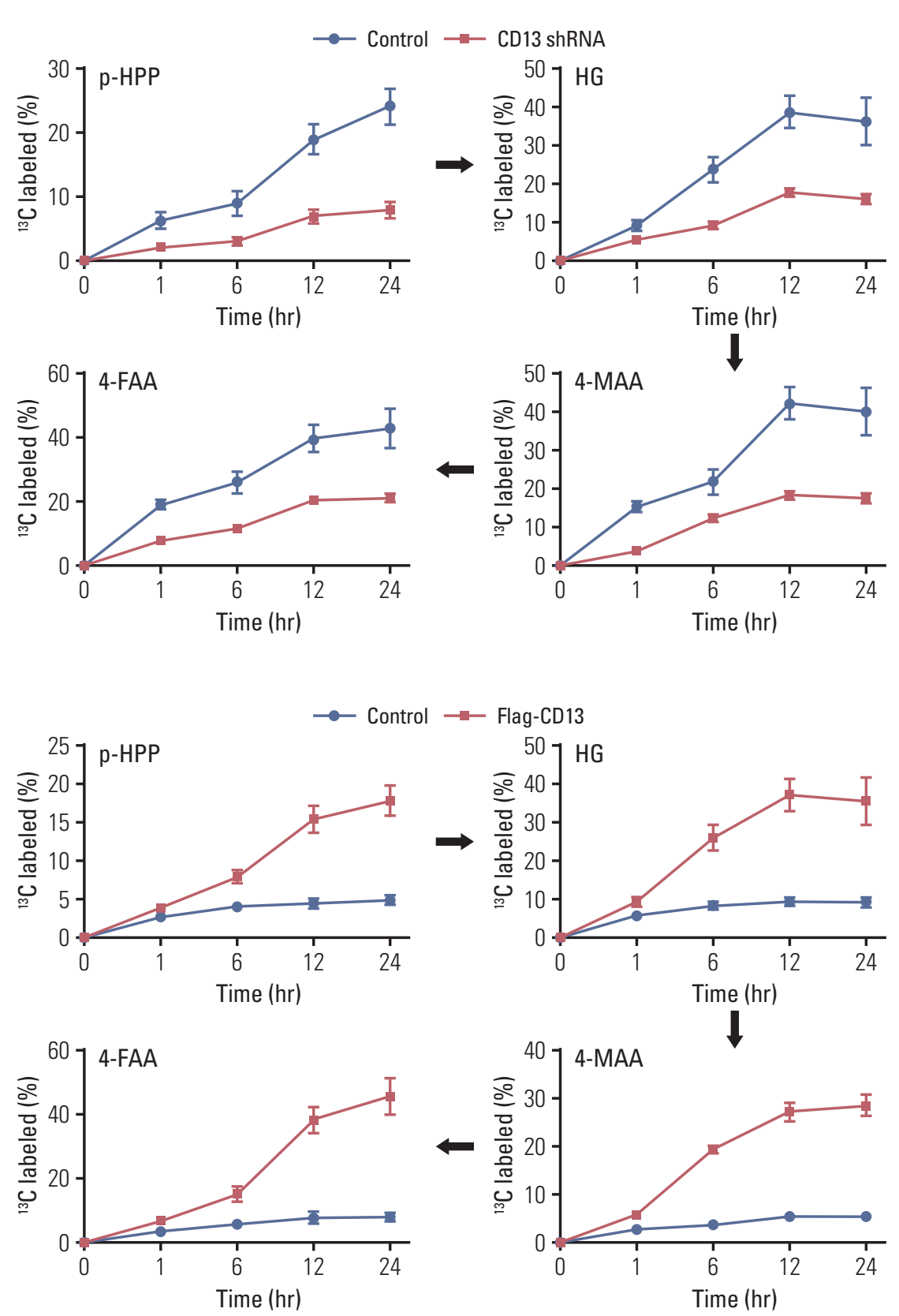

Fig. 2. (Continued from the previous page) (E) The percentage of ${ }^{13} \mathrm{C}$-labeled intermediates (p-HPP, HG, 4-MAA, and 4-FAA) in HuH7-derived $\mathrm{CD}_{13}{ }^{+}$cells pre-treated with CD13 shRNA or scrambled shRNA. (F) The percentage of ${ }^{13} \mathrm{C}-$ labeled intermediates in HuH7-derived $\mathrm{CD}^{+} 3^{+}$cells overexpressing Flag-CD13. (Continued to the next page)

(Fig. 1A right and B). The quiescent state of CSCs is believed to protect them from therapeutic agents [14]. As compared with $\mathrm{CD} 13^{-}$cells, the matched $\mathrm{CD} 13^{+}$subpopulation expressed lower levels of markers for cell proliferation, including Ki67, proliferating cell nuclear antigen, and MCM2 (Fig. 1C). Analysis of HepG2 and HuH7 cells using Hoechst plus pyro-
ninY staining showed that $>60 \%$ of $\mathrm{CD}^{+} 3^{+}$fraction were in the $\mathrm{G} 0$ phase. In contrast, most CD13- fractions were actively cell cycling ( $<40 \%$ in the G0 phase) (Fig. 1D). In addition, we examined changes in CD13 staining in nine HCC patients after receiving hepatic arterial infusion chemotherapy, using low-dose concomitant radiochemotherapy with cisplatin 


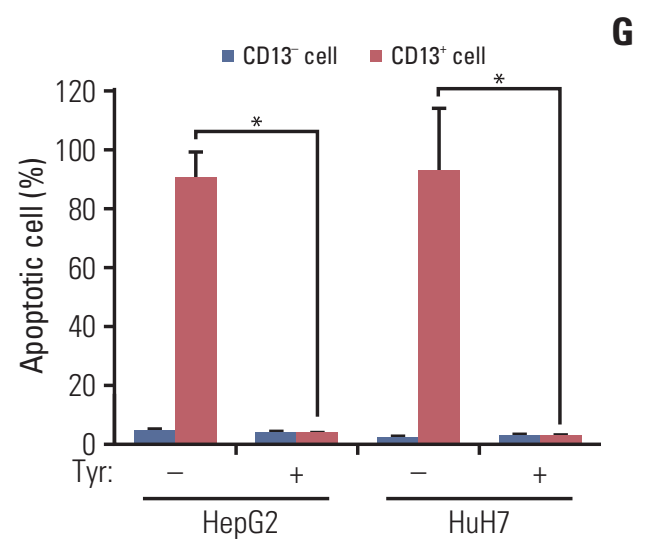

Fig. 2. (Continued from the previous page) $(\mathrm{G})$ The apoptotic rate in $\mathrm{CD}_{13}{ }^{+}$and $\mathrm{CD}_{13}{ }^{-}$fractions from HepG2 and $\mathrm{HuH7}$ cells upon Tyr deprivation for 72 hours. Values shown are mean \pm standard deviation. A two-tailed unpaired $t$ test was used to compare experimental groups. ${ }^{*} \mathrm{p}<0.05$.

(CDDP) and 5-FU with/ without leucovorin. All patients initially achieved a partial regression to drug treatment. As compared to the pretreatment values, the score of CD13 staining was significantly increased in seven out of nine residual tumors (Fig. 1E and $\mathrm{F}$ ).

Three-dimensional (3D) organoids serve as an excellent tool to recapitulate tumorigenic aspects and drug responses of advanced human carcinomas [15]. In the current study, we generated 3D organoids from therapy-naive patients with HCC. Briefly, single-cell suspensions from tumor biopsies were suspended in Matrigel with fully supplemented aDM$\mathrm{EM} / \mathrm{F}^{2} 2^{+}$. Spherical structures, which were defined as 3D organoids, were detected by day 7 and continued to grow to $\sim 200 \mu \mathrm{m}$ in diameter by day 14 (Fig. 1G). We successfully generated 3D organoids from three of five tumors $(60 \%)$, two (T3 and T4) of which could be passaged at least once to exhibit a continuous growth (Fig. 1H). Quantitative analysis demonstrated that the organoids harbored similar percentages of $\mathrm{CD}^{2} 3^{+}$cells when compared with the corresponding primary tumors (Fig. 1I). Moreover, the proportion of $\mathrm{CD} 13^{+}$ cells remained relatively constant in organoids during serial passages (Fig. 1J), suggesting that organoids contain steady ratios of CSC populations in long-term cultures. Next, we evaluated the efficiency of organoid formation after treatment of chemotherapeutic agents. Upon administration of 5-FU or sorafenib, the efficiency of organoid formation decreased; this impairment remained unchanged along with serial passages of organoid culture (Fig. 1K). More importantly, the frequency of $\mathrm{CD}^{+} 3^{+}$cells, as detected by fluorescence-activated cell sorting, was significantly increased in organoids following treatments of chemotherapeutic agents
(Fig. 1L). Together, these data imply a universal chemoresistance of $\mathrm{CD}^{+} 3^{+} \mathrm{CSC}$ s in HCCs.

\section{Enhancement of Tyr metabolism in $\mathrm{CD}_{13}{ }^{+} \mathrm{CSC}$ from HCCs}

In comparison with differentiated tumor cells, CSCs are more dependent on glycolysis [16]. Unexpectedly, CD13 ${ }^{+}$ cells had negligible glycolytic activity than the matched CD13- counterpart (S1A Fig.). Glucose and glutamine are essential energy sources for cancer cells [17]. Unlike CD13cells (S1B Fig.), however, deprivation of glucose or glutamine did not affect the viability of $\mathrm{CD} 13^{+}$fraction (Fig. 2A), suggesting a switch to alternative nutrient supply. Removing essential nutrients, one at a time, revealed significant apoptosis upon deprivation of leucine, valine, and most notably tyrosine (Fig. 2A). Deprivation of phenylalanine, a precursor of Tyr, did not affect cell survival (Fig. 2A), due to deficiency of phenylalanine hydroxylase in these cells. A significant reduction of Tyr concentration in culture medium during 24 hours incubation was observed in $\mathrm{CD}^{2} 3^{+}$but not $\mathrm{CD}^{-} 3^{-}$cells (Fig. 2B). Tyr consumption by $\mathrm{CD}_{13}{ }^{+}$cells was accompanied by higher expression of enzymes in Tyr degradation (tyrosine aminotransferase [TAT], 4-hydroxyphenylpyruvic acid dioxygenase [HPD], homogentisate 1,2-dioxygenase [HGD], maleylacetoacetate isomerase [MAAI], and fumarylacetoacetase [FAH]) (Fig. 2C). Furthermore, we infused ${ }^{13} \mathrm{C}$-Tyr into HepG2-xenografted nude mice and examined ${ }^{13} \mathrm{C}$ label incorporation into Tyr metabolites (para-hydroxyphenylpyruvate, homogentisic acid, 4-maleylacetoacetate, 4-fumarylacetoacetate [4-FAA]) (S1C Fig.) in CD13 ${ }^{+}$cells. As shown in Fig. 2D, Tyr tracing experiments in HepG2 xeno-grafts demonstrated higher flux through Tyr degradation pathway in $\mathrm{CD}_{13}^{+}$than in $\mathrm{CD}^{-} 3^{-}$cells.

The differential dependence on Tyr metabolism between $\mathrm{CD}^{+}{ }^{+}$and $\mathrm{CD}^{-} 3^{-}$cells prompts us to explore the underlying mechanism(s). We examined whether CD13 is the key determinant for activation of tyrosine metabolism in $\mathrm{CD}_{13}{ }^{+} \mathrm{CSC}$. Notably, metabolomic analysis using ${ }^{13} \mathrm{C}$-Tyr tracing revealed that CD13 knockdown decreased Tyr uptake (S1D Fig.), attenuated the flux of intermediates into Tyr degradation (Fig. 2E) and increased glycolytic rate (S1E Fig.). On the contrary, overexpression of CD13 in CD13- cells recapitulated the metabolite phenotype observed in the $\mathrm{CD}_{13}{ }^{+}$counterpart (Fig. 2F, S1F and S1G Fig.), highlighting the specificity of CD13 for metabolic switch in $\mathrm{CD}_{13}{ }^{+}$subpopulation. Consistently, Tyr deprivation led to massive apoptosis in cultured $\mathrm{CD}^{+}{ }^{+}$cells and spared $>95 \% \mathrm{CD}^{-}$fraction (Fig. 2G). 
A
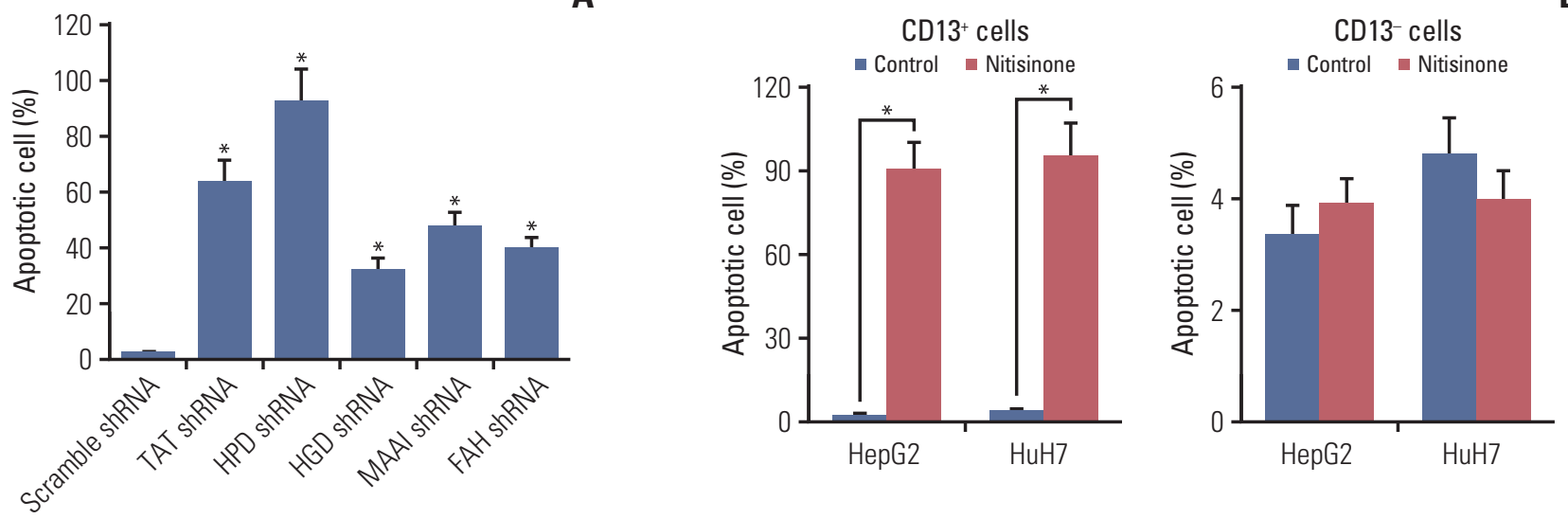

C
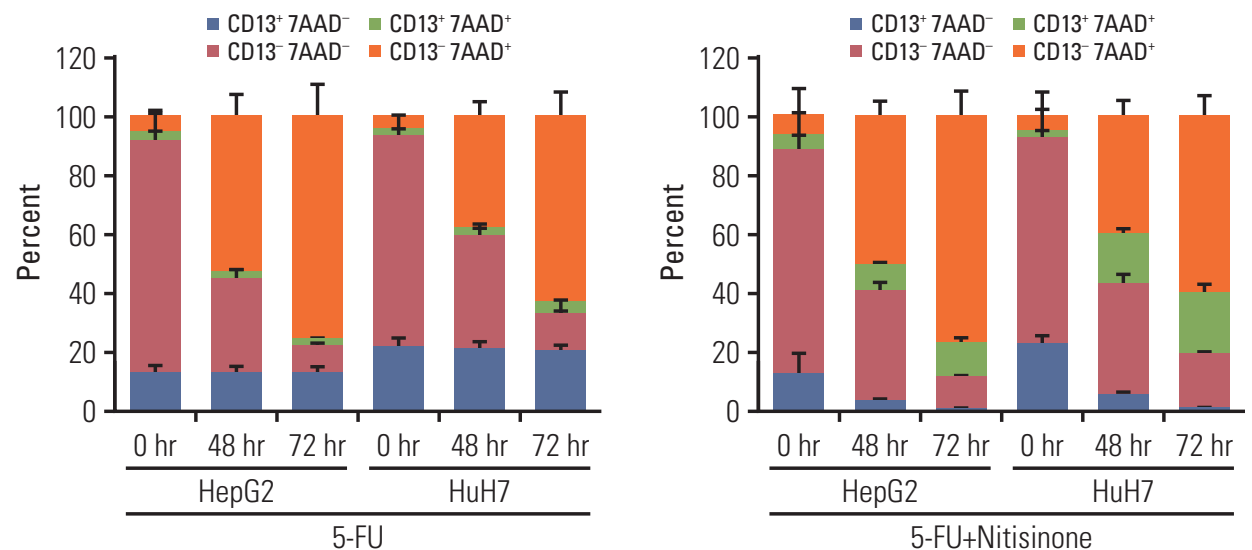

Fig. 3. Targeting Tyr metabolism by nitisinone eliminates $\mathrm{CD} 13^{+}$cancer stem cells and postpones recurrence in hepatocellular carcinomas. (A) The apoptotic rate in HepG2-derived $\mathrm{CD} 13^{+}$cells transfected with the indicated shRNA for 72 hours. TAT, tyrosine aminotransferase; HPD, 4-hydroxyphenylpyruvic acid dioxygenase; HGD, homogentisate 1,2-dioxygenase; MAAI, maleylacetoacetate isomerase; FAH, fumarylacetoacetase. (B) The apoptotic rate in HepG2 and HuH7-derived CD13 ${ }^{+}$(left panel) versus CD13- subsets (right panel) with or without nitisinone (100 nM, 72 hours) treatment. (C) HepG2 and HuH7 cells were persistently treated with 5-fluorouracil (5-FU; $5 \mu \mathrm{M})$ or / and nitisinone (100 nM), after which CD13 expression was analyzed by flow cytometry at the indicated time points. Dead cells were detected by 7AAD staining. (Continued to the next page)

\section{Ablating $\mathrm{CD}^{+} 3^{+} \mathrm{CSC}$ and postponing recurrence in HCCs by nitisinone via targeting Tyr metabolism}

Since CD13+ CSCs in HCCs are Tyr addictive, we examined whether Tyr metabolism is an effective target for preventing tumor recurrence. As almost all enzymes in Tyr degradation were found differentially regulated in $\mathrm{CD}_{13}{ }^{+}$ CSCs, we explored shRNAs against TAT, HPD, HGD, MAAI, and FAH in a series of preliminary cytotoxicity assays. Here, shRNA against HPD revealed the most prominent results (Fig. 3A); thus, HPD was selected for subsequent studies. Nitisinone, a commercial safe drug licensed for treatment of hereditary tyrosinaemia, can interrupt tyrosine metabolism through inhibition of HPD [18]; it was prioritized for followup. Firstly, we evaluated the in vitro sensitivity of $\mathrm{CD} 13^{+}$cells to nitisinone. Cytotoxicity assays confirmed a dramatic targeting of $\mathrm{CD}_{13}{ }^{+}$cells with much less effect in $\mathrm{CD}^{-} 3^{-}$compartment (Fig. 3B). Flow cytometric quantitation of Hep3G and $\mathrm{HuH} 7$ cells revealed a complete loss of $\mathrm{CD}_{13}{ }^{+}$cells under nitisinone treatment, overcoming the 5-FU-induced enrichment of this fraction (Fig. 3C). The tumor-suppressive potential of ablating $\mathrm{CD}_{1} 3^{+} \mathrm{CSCs}$ was further demonstrated in 3D collagen-embedded spheroids of Hep3G cells, a model which more readily mimics the hepatic carcinoma microenviron- 
D

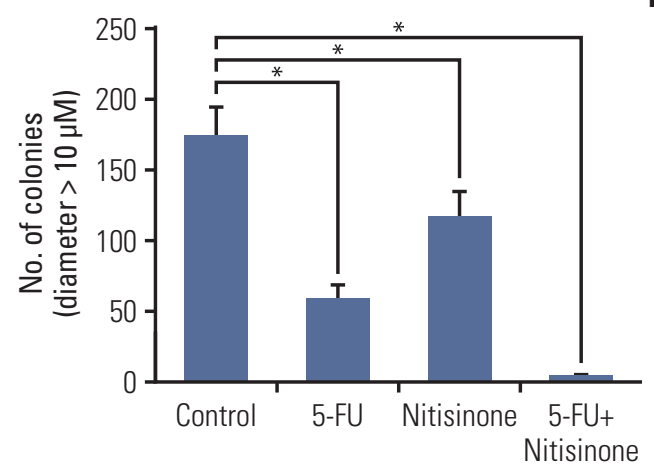

E

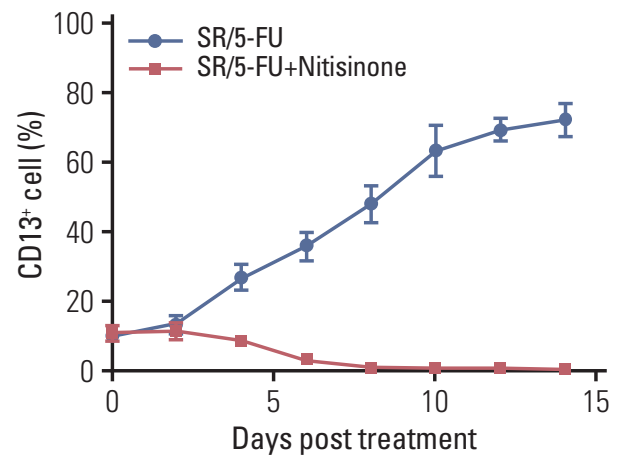

G
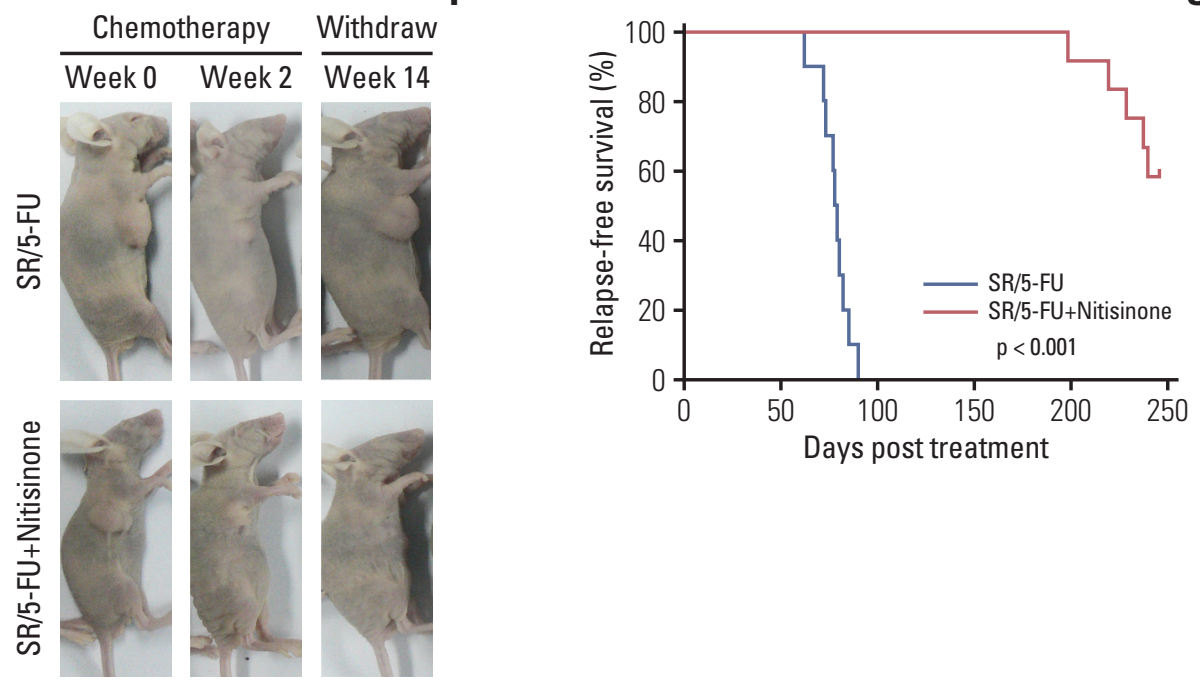

Fig. 3. (Continued from the previous page) (D) Soft agar colony-forming assay of HepG2 cells after 72 hours pulse treatment with 5-FU $(5 \mu \mathrm{M})$ or / and nitisinone $(100 \mathrm{nM})$. Colonies were counted manually after 5 weeks of growth. (E-G) Upon reaching $\sim 1 \mathrm{~cm}$ in tumor diameter after subcutaneous implantation, BALB/c nude mice bearing HepG2 xenografts were randomized to receive sorafenib (SR)/5-FU (twice weekly for 2 weeks) in the presence $(n=20)$ or absence $(n=20)$ of nitisinone. (E) The frequency of $\mathrm{CD}^{+} 3^{+}$cell between SR/5-FU and SR/5-FU+nitisinone groups was compared by flow cytometry ( $\mathrm{n}=3$ per time point). (F) Macroscopic examination revealed regression within 2 weeks (middle panels), and subsequent relapse or complete regression within 14 weeks (right panels) in SR/5-FU and SR/5-FU+nitisinone groups, respectively. (G) Relapse-free survival was compared between doxorubicin (DXR)/5-FU and DXR/5-FU plus nitisinone groups by Kaplan-Meier survival analyses. Values shown are mean \pm standard deviation. p-values were calculated by two-tailed unpaired t test unless otherwise indicated. ${ }^{*} \mathrm{p}<0.05$.

ment. Treatment of Hep3G cells with a single pulse of nitisinone plus 5-FU over 72 hours substantially reduced longterm growth in soft agar and the number of 3D colonies remained at almost undetectable levels, even after 5 weeks of culture in drug-free medium (Fig. 3D). These results indicate that even a relatively short-term treatment can yield a sustained anti-tumor effect.

Nitisinone treatment also improved the tumor-suppressive potential of chemotherapy in vivo. HepG2 cells were subcu- taneously transplanted into BALB/c nude mice, and were allowed to grow until tumor reached a comparable size prior to receiving doxorubicin/5-FU regimen with or without nitisinone. Nitisinone alone gradually eliminated $\mathrm{CD}^{+} 3^{+}$cells within 8 days (Fig. 3E). Consequently, the combined treatment significantly postponed the time to recurrence (by $>21$ weeks vs. mice receiving FOLFOX alone) (Fig. 3F and G). 
A
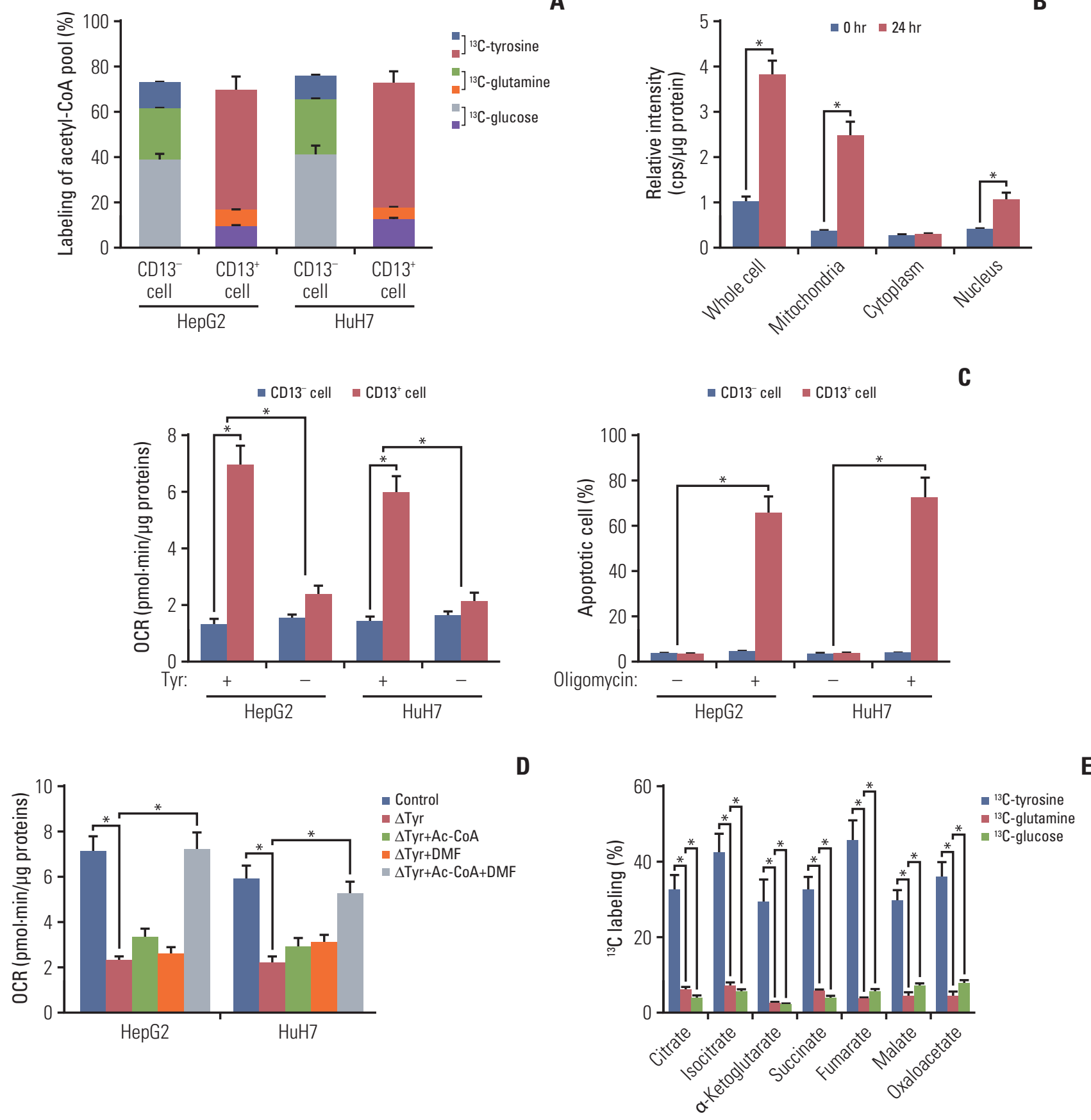

Fig. 4. Tyr metabolism provides mitochondrial acetyl-CoA for oxidative phosphorylation. (A) Acetyl-CoA from ${ }^{13} \mathrm{C}$-glucose, ${ }^{13} \mathrm{C}$-glutamine, or ${ }^{13} \mathrm{C}$-Tyr in $\mathrm{CD} 13^{+}$and $\mathrm{CD} 13^{-}$fractions sorted from HepG2 and $\mathrm{HuH} 7$ cells. (B) The intracellular distribution of ${ }^{13} \mathrm{C}$-acetyl-CoA in HepG2-derived $\mathrm{CD}_{13}{ }^{+}$cells incubated with ${ }^{13} \mathrm{C}$-tyrosine for 24 hours. (C) Upper panel: oxygen consumption of HepG2 and HuH7-derived $\mathrm{CD}^{+} 3^{+}$and $\mathrm{CD}^{-} 3^{-}$cells cultured in complete or Tyr-deprived medium for 24 hours; lower panel: apoptotic rate of HepG2 and HuH7-derived CD13 ${ }^{+}$and CD13- cells cultured with oligomycin $(2.5 \mu \mathrm{g} / \mathrm{mL})$ for 72 hours. (D) Oxygen consumption of HepG2 and HuH7-derived $\mathrm{CD}_{13}{ }^{+}$cells cultured in Tyr-deprived medium and pretreated with $200 \mu \mathrm{M}$ acetyl-CoA, $50 \mu \mathrm{M}$ dimethyl-fumarate (DMF), or both for 24 hours. (E) HepG2-xenografted BALB/c nude mice were randomized to receive ${ }^{13} \mathrm{C}$-glucose $(\mathrm{n}=5),{ }^{13} \mathrm{C}$-glutamine $(\mathrm{n}=5)$, or ${ }^{13} \mathrm{C}$-tyrosine $(\mathrm{n}=6)$ infusion for 200 minutes prior to cytometric sorting of $\mathrm{CD}_{13}{ }^{+}$cells. The percentage of ${ }^{13} \mathrm{C}$-labeled TCA cycle intermediates was assessed by mass spectrometry. (Continued to the next page) 
$\mathbf{F}$
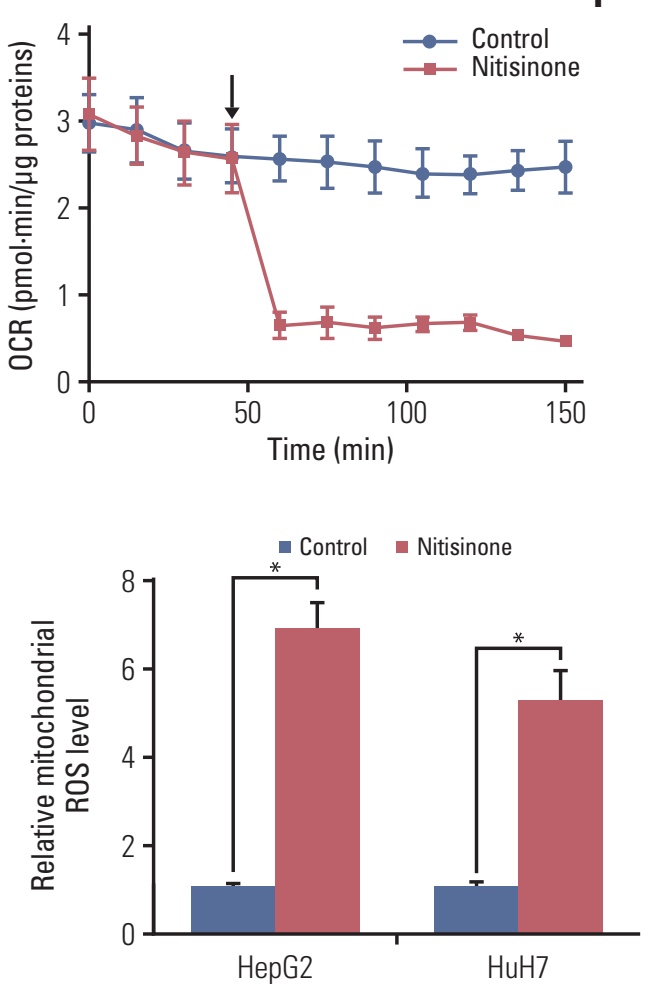

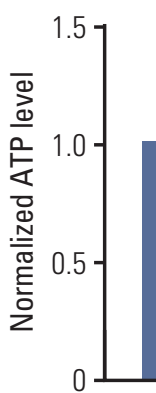

* Control Nitisinone

G

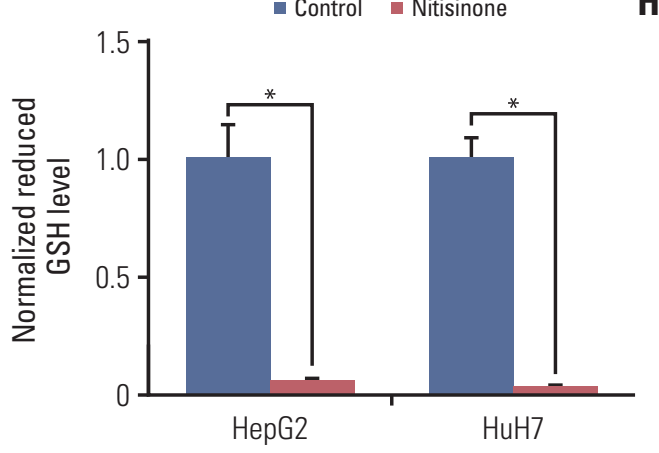

Fig. 4. (Continued from the previous page) (F) Plots of oxygen consumption rate as a parameter of time in the absence or presence of $100 \mathrm{nM}$ Nitisinone (indicated by arrow) in CD13 ${ }^{+}$fraction sorted from HuH7 cells. (G) ATP levels in HepG2 and HuH7derived $\mathrm{CD}_{13}{ }^{+}$fraction treated with or without nitisinone $(100 \mathrm{nM})$ for 6 hours. $(\mathrm{H})$ Mitochondrial reactive oxygen species (ROS; left panel) and reduced glutathione (GSH) levels (right panel) in HepG2 and HuH7-derived CD13 ${ }^{+}$cells subjected to $100 \mathrm{nM}$ nitisinone for 6 hours. Values shown are mean \pm standard deviation. A two-tailed unpaired $\mathrm{t}$ test was used to compare experimental groups. ${ }^{*} \mathrm{p}<0.05$.

\section{Entry of Tyr-derived mitochondrial acetyl-CoA into TCA cycle}

In Tyr degradation pathway, 4-FAA is catabolized by FAH to yield end-products, fumarate and acetoacetate, after which the latter is converted into acetyl-CoA (S1C Fig.). In vitro experiments using ${ }^{13} \mathrm{C}$-labeled Tyr showed $\sim 53 \%$ contribution of the acetyl-CoA pool by Tyr in $\mathrm{CD}^{+} 3^{+}$cells but negligible contribution of Tyr in $\mathrm{CD}^{-}{ }^{-}$cells (Fig. 4A). In contrast, experiments using ${ }^{13} \mathrm{C}$-glucose or ${ }^{13} \mathrm{C}$-glutamine revealed $<20 \%$ contribution to the acetyl-CoA pool in $\mathrm{CD}^{+} 3^{+}$cells (Fig. 4A). Furthermore, targeted metabolomic analysis using 13C-labeled Tyr showed increased Tyr-derived 4-FAA (and total 4-FAA) and decreased acetyl-CoA (and total acetylCoA) by FAH knockdown (S2A and S2B Fig.), again suggesting Tyr contributes to the maintenance of the acetyl-CoA pool in $\mathrm{CD}_{13}^{+}$cells.

Acetyl-CoA from Tyr was present in both mitochondria and nucleus (Fig. 4B). Mitochondrial acetyl-CoA enters into the TCA cycle for generation of energy. TCA activity in $\mathrm{CD}^{+}{ }^{+}$cells, as reflected by oxygen consumption rate (OCR), was 3.5-fold higher than in the matched CD13- cells; deprivation of Tyr, but not other essential nutrients, eliminated such a difference (Fig. 4C left, S2C Fig.). The importance of oxidative phosphorylation was further demonstrated by increased apoptosis upon treatment with the mitochondrial ATP synthase inhibitor oligomycin (Fig. 4C right). Of note, exogenous acetyl-CoA or cell-permeable dimethyl-fumarate (DMF) alone did not restore OCR upon Tyr deprivation, whereas combination of acetyl-CoA and DMF dramatically rescued OCR in $\mathrm{CD}_{13}{ }^{+}$cells (Fig. 4D), indicating that Tyr yields acetyl-CoA and fumarate to support TCA cycle. In line with these, Tyr is the major carbon source for the TCA cycle in $\mathrm{CD}_{13}{ }^{+}$CSCs, as determined by in vivo infusing HepG2xenografted mice with uniformly labeled ${ }^{13} \mathrm{C}$-glucose, ${ }^{13} \mathrm{C}$ glutamine, and ${ }^{13} \mathrm{C}$-tyrosine, respectively (Fig. 4E).

We further determined the bioenergetic properties of $\mathrm{CD}^{+}{ }^{+} \mathrm{CSC}$ s treated with nitisinone. Nitisinone inhibited 
A

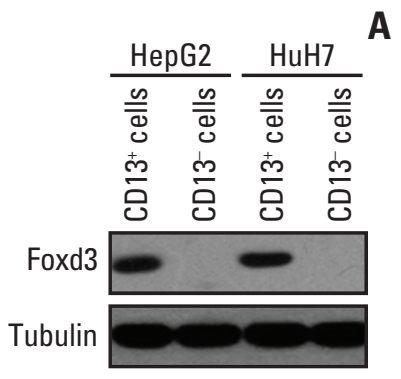

B

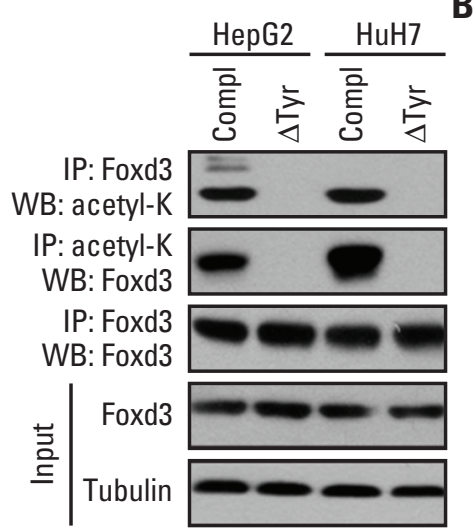

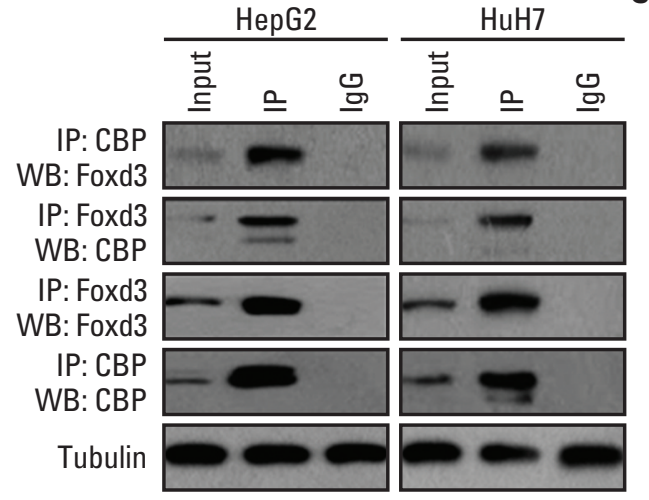
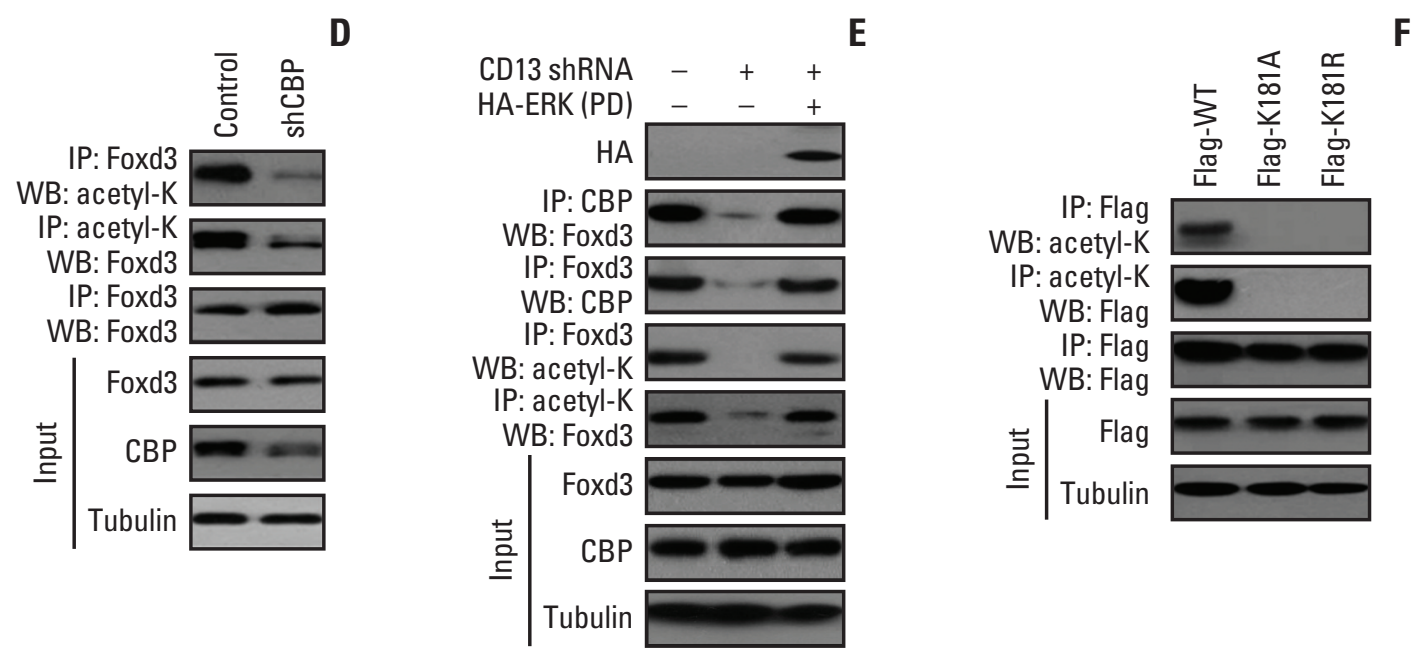

E 

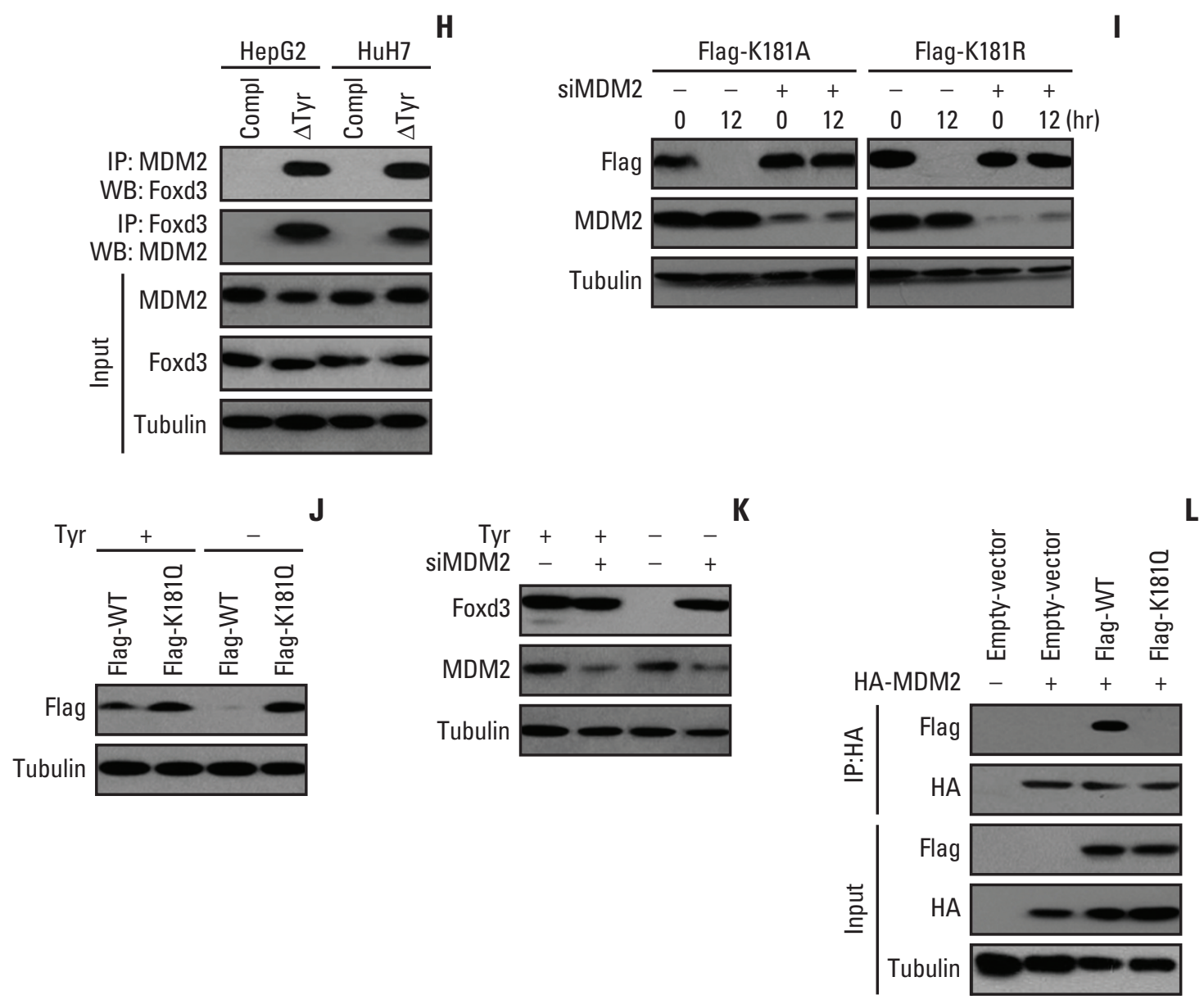

Fig. 5. (Continued from the previous page) $(\mathrm{H}) \mathrm{Co}-\mathrm{IP}$ of Foxd 3 and MDM2 in $\mathrm{CD} 13^{+}$cells after Tyr deprivation for 6 hours. (I) The expression of K181A/R mutants and MDM2 in HepG2-derived CD13+ cells transfected with K181A/R, MDM2 siRNA, or both. The time points are presented using 36 hours after transfection as the reference. (J) The expression of wild-type Foxd3 and K181Q mutant in HuH7-derived CD13+ cells after Tyr deprivation for 12 hours. (K) HuH7-derived CD13 ${ }^{+}$cells were treated with or without MDM2 siRNA, after which Foxd3 and MDM2 expression were assessed at 12 hours after Tyr deprivation. (L) Co-IP of HA-tagged MDM2 and Flag-tagged wild-type Foxd3 or K181Q in HEK293T cells treated with MG132 (25 nM).

nitisinone induced mitochondrial dysfunction and energy depletion in $\mathrm{CD}_{13}{ }^{+} \mathrm{CSC}$, we asked if oxidation was promoted. Nitisinone induced a clear upregulation of mitochondrial reactive oxygen species (ROS) in $\mathrm{CD}^{+} 3^{+}$cells, accompanied with decreased levels of reduced glutathione (Fig. 4H). Therefore, HPD inhibition by nitisinone severely impaired redox balance and energy control in $\mathrm{CD}_{13}{ }^{+} \mathrm{CSC}$, thereby eliminating this fraction.

\section{Prevention of Foxd3 degradation by Tyr-derived nuclear acetyl-CoA}

Nanoflow liquid chromatography-mass spectrometry analysis indicated that nuclear acetyl-CoA produced by Tyr metabolism does not contribute acetyl group for histone acetylation in $\mathrm{CD}^{+}{ }^{+}$cells (S3A Fig.). Spiking CD13 ${ }^{+}$cells with ${ }^{13} \mathrm{C}$-Tyr followed by immunoprecipitating acetylated peptides from nuclear extracts resulted in 14 nuclear proteins containing ${ }^{13} \mathrm{C}$-acetyllysine (S4 Table). No ${ }^{13} \mathrm{C}$-acetyl peptides corresponding to known acetylation sites on core histones were discovered. Notably, Foxd3 knockdown impaired the resistance of $\mathrm{CD} 13^{+}$cells to $5-\mathrm{FU}$ or SR to a greater extent than with knockdown of any other candidate proteins (S3B Fig.). Foxd3 was preferentially expressed in $\mathrm{CD} 13^{+}$fraction, but not in the matched CD13- cells (Fig. 5A), at least partially explaining the therapeutic vulnerability of $\mathrm{CD} 13^{+}$cells. Therefore, we focused on Foxd3 in subsequent studies. Foxd3 acetylation was detectable in $\mathrm{CD}^{+} 3^{+}$cells (Fig. 5B). Tyr dep- 

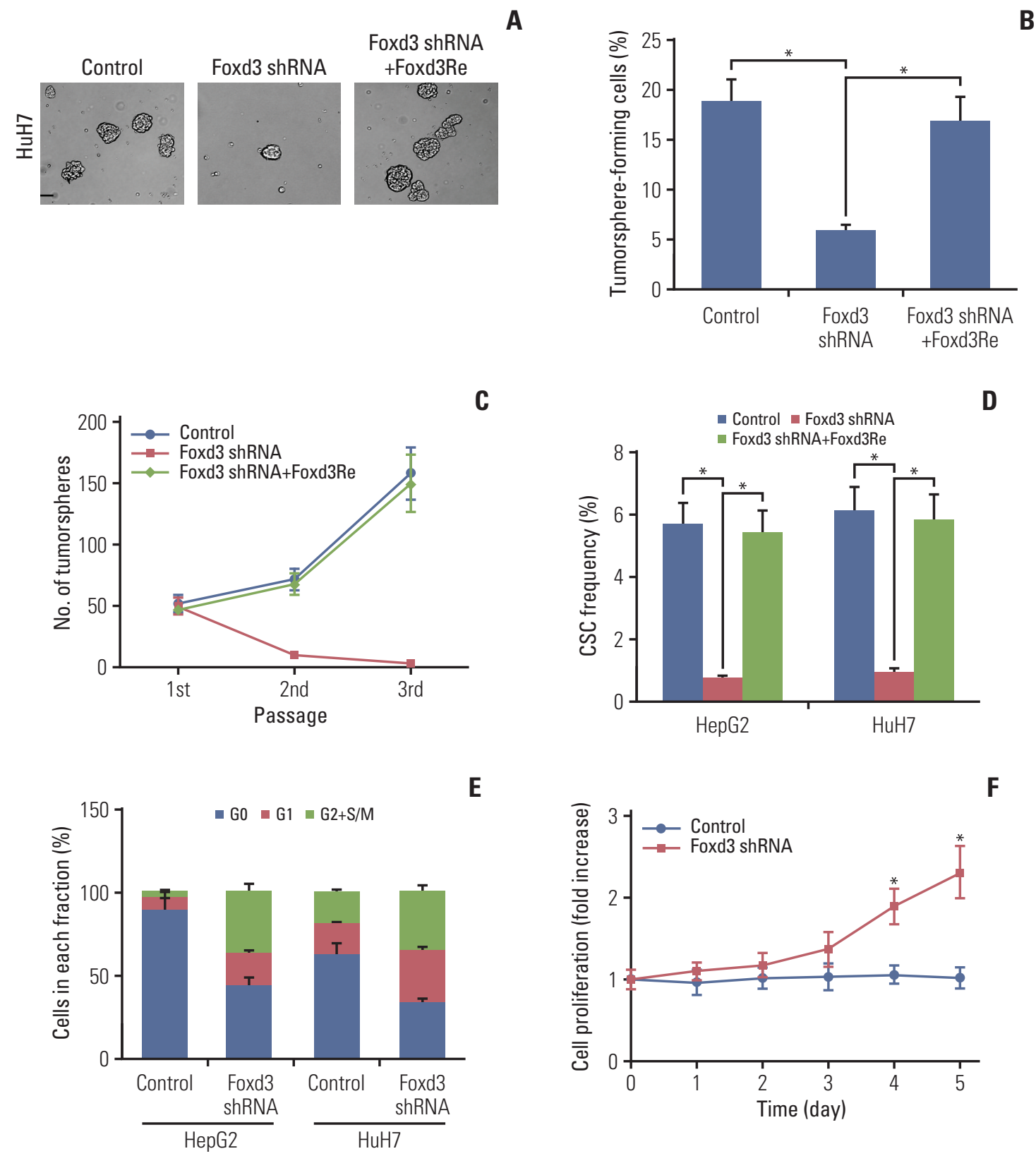

E

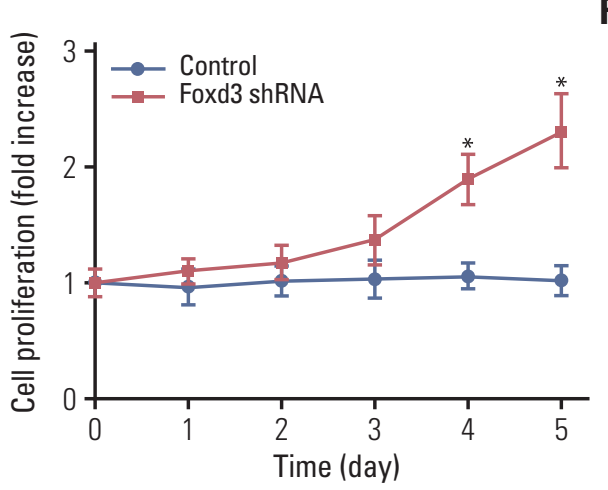

Fig. 6. Loss of Foxd3 impairs self-renewal and quiescence in CD13+ cancer stem cells (CSCs). (A) Sphere formation by HepG2derived $\mathrm{CD}_{13}{ }^{+}$cells expressing Foxd3 shRNA or/and Foxd3Re. Tumorsphere-forming capacity (B) and serial spheres passaging (C) of HepG2-derived CD13 ${ }^{+}$cells expressing Foxd3 shRNA and/or Foxd3Re. (D) HepG2 and HuH7-derived CD13 ${ }^{+}$ cells were transfected with Foxd3 shRNA alone or in combination with Foxd3Re, after which CSC frequency of CD13 ${ }^{+}$CSCs was measured by in vivo limiting dilution assays. (E) Cell cycle distribution of HepG2 and HuH7-derived CD13 $3^{+}$cells expressing scrambled shRNA or Foxd3 shRNA. (F) The relative proliferative rate of HuH7-derived $\mathrm{CD}_{13}{ }^{+}$cells on 5 days upon Foxd3 knockdown. Values shown are mean \pm standard deviation. A two-tailed unpaired $t$ test was used to compare experimental groups. ${ }^{*} \mathrm{p}<0.05$.

rivation in culture medium abrogated Foxd3 acetylation (Fig. 5B). Also, Foxd3 was no longer acetylated in a TAT or FAH mutant (S3C Fig.). To identify the acetyltransferase res- ponsible for Foxd3 acetylation, we knocked down 12 lysine acetyltransferases with siRNA (S3D Fig.), one at a time, and found that only CBP regulated Foxd3 acetylation (S3E Fig.). 
Immunoprecipitation further revealed association between CBP and Foxd3 (Fig. 5C). Transduction of $\mathrm{CD}^{3} 3^{+}$cells with shRNA targeting CBP abrogated Foxd3 acetylation (Fig. 5D). Previous studies reported that mitogen-activated protein kinase (MAPK) / extracellular signal-regulated kinase (ERK) kinases could increase CBP acetyltransferase activity by phosphorylating CBP at its C-terminal [19]. Since signaling via ERK1/2 lies downstream of the CD13/MAPK axis [20], treatment of $\mathrm{CD}_{13}{ }^{+}$cells with $\mathrm{CD} 13$ shRNA decreased the level of the CBP-Foxd3 complex and blocked Foxd3 acetylation (Fig. 5E), which were largely rescued by a constitutively active ERK mutant (Fig. 5E). These data suggested that ERK1/2 signaling is required for CBP-Foxd3 interaction and Foxd3 acetylation.

Tandem mass spectrometry analysis of Flag-tagged Foxd3 identified an acetylation site at K181 (S3F Fig.). Acetylation was evident in $\mathrm{CD}_{13}{ }^{+}$cells with wild-type Foxd3 (wtFoxd3) but not the acetylation-resistant K181A or K181R mutant (Fig. 5F). Incidentally, both K181 mutants were subjected to degradation and became undetectable within 12 hours of ectopic expression (Fig. 5G). Treatment with the proteasomal inhibitor MG132 increased the stability of K181 mutants (Fig. $5 G$ ), indicating that these mutants are degraded through the ubiquitin-proteosome system. ERK activation leads to FOXO proteins ubiquitination mediated by MDM2 E3 ligase [21]. We found that endogenous Foxd3 binds to MDM2 upon Tyr deprivation (Fig. 5H). After transfection of the K181A or K181R mutant, MDM2 interacted with these mutants (S3G Fig.). As expected, knockdown of MDM2 decreased turnover of K181A or K181R proteins (Fig. 5I), suggesting that K181 acetylation protects Foxd3 from MDM2-mediated degradation. In contrast, we observed undetectable level of wtFoxd3, but high level of constitutively acetylated K181Q mutant under Tyr-free conditions (Fig. 5J). MDM2 knockdown also rescued degradation of endogenous Foxd3 upon Tyr deprivation (Fig. 5K), without affecting Foxd3 mRNA (S3H Fig.), suggesting that Foxd3 expression is regulated at the posttranscriptional level. Consistently, immunoprecipitation analysis revealed an association of HA-tagged MDM2 with wtFoxd3 in HEK293T cells (due to negligible endogenous CBP activity), but not with K181Q (Fig. 5L).

\section{Impairment of self-renewal and quiescence of $\mathrm{CD} 13^{+}$ cells by loss of Foxd3}

Next, we examined the role of Foxd3 in the maintenance of $\mathrm{CD}_{13}{ }^{+} \mathrm{CSC}$. In a tumorsphere experiment, Foxd3 knockdown (Foxd3KD) with an shRNA (S5A Fig.) could impair self-renewal of $\mathrm{CD} 13^{+}$cells, as reflected by significantly reduced tumorspheres formation (Fig. 6A and B) and decreased passaging and expansion (Fig. 6C). Next, we gave mice limiting doses of Foxd3KD or control CD13 ${ }^{+}$cells. Tumors gen- erated from a single cell (with limiting dilution) were transplanted into secondary recipients. The results demonstrated that Foxd3KD decreased the frequency of self-renewing cells by $>8$-folds (Fig. 6D). These effects were largely rescued by a human Foxd3 plasmid (Foxd3Re) resistant to Foxd3KD (Fig. 6A-D). Sorted $\mathrm{CD}_{13}{ }^{+}$cells did not show increased propidium iodide (PI) uptake upon Foxd3KD (S5B Fig.), thereby excluding the possibility that the pool of $\mathrm{CD}_{1} 3^{+}$cells was exhausted by a process of cell death. Quiescence protects stem cells against loss of self-renewal capacities [22]. In our experiments, Hoechst/ PyroninY staining revealed that Foxd$3 \mathrm{KD}$ in $\mathrm{CD} 13^{+}$cells decreased the percentage of cells in the G0 phase, and increased the proportion in G1/S/G2M phases (Fig. 6E). Accordingly, $\mathrm{CD}^{+} 3^{+}$cells proliferated faster after Foxd3KD (Fig. 6F). These data indicated that increased proliferation likely contributes to the reduced self-renewal of Foxd3KD cells by diminishing the pool of quiescent stem cells.

\section{Discussion}

Most tumor therapies, including chemo- or targeted-therapies and ionizing radiation, preferentially affect cycling cells but spare quiescent cells. Here we showed that functionally defined $\mathrm{CD}_{13}{ }^{+} \mathrm{CSC}$ in $\mathrm{HCC}$ are characterized as quiescent and do not respond to chemotherapeutic agents. Drug sensitivity is, therefore, a functional property that varies between the heterogeneous cell types comprising HCCs. As stem cell signature expression in various tumor correlates inversely with outcome [23], we proposed that the degree of CD13 at the gene or protein level relates to the size of the clinically essential pool that can cause relapse.

One strategy towards eliminating quiescent cancer cells is to selectively target unique metabolic demands of these cells [24]. In addition to changes in the metabolism of glucose and glutamine, the role of amino acids in supporting tumor growth has also been increasingly implicated. Using in vivo negativeselection RNAi screens, Possemato et al. [25] established that the serine pathway is essential to most estrogen negative breast cancers subtypes. The apoptotic pathways engaged by amino acid shortage have not been thoroughly explored, but some reports indicate that cell death occurs via mitochondrial apoptosis, possibly mediated by the Noxa/Mcl-1 axis [26]. In this study, the quiescent phenotype of $\mathrm{CD}_{13}{ }^{+} \mathrm{CSC}$ s reflected a metabolic state that is characterized by higher dependence on exogenous Tyr to survive relative to the rapidly proliferating tumor bulk population. This dependence was attributed to the presence of CD13-induced signaling cascade, which constituted a unique mechanism to regulate the 
metabolic switch from glycolysis to Tyr degradation. Tyrosine, a non-essential amino acid, can be degraded into acetyl$\mathrm{CoA}$ and fumarate to support citric acid cycle. Thus, $\mathrm{CD} 13^{+}$ CSCs depend on mitochondrial function, but not glycolysis, to meet their energy demands. As mitochondrial respiration is the major cellular process that produces ROS, our findings converge on previous reports that $\mathrm{CD} 13^{+} \mathrm{CSC}$ s have relatively higher ROS than in their CD13- counterparts $[8,27]$.

Apart from its central role in metabolism, acetyl-CoA regulates cell functions by participating in the acetylation of a variety of proteins [28]. The current study further proved that Tyr metabolism produces acetyl-CoA in the nuclear compartment to acetylate a number of non-histone proteins, including Foxd3, a transcriptional factor belonging to the Fox family. Foxd 3 is expressed in dorsal and paraxial mesoderm and neural crest, as well as inner cell mass and trophectoderm of mammalian embryonic stem cells (ESCs). Foxd3 is involved in pluripotency maintenance in human ESCs, in promoting epithelial to mesenchymal transition during neural crest specification, in regulating the balance between melanocyte and Schwann cell development, and in regulation of a set of differentiation-associated genes along with NFATc3 in ESCs [29]. In the context of mutated Ras-driven tumors, activation of ERK leads to phosphorylation and subsequent degradation of FOXO proteins in an MDM2-dependent manner [21]. However, it appears that this is not the case in $\mathrm{CD}_{13}{ }^{+} \mathrm{CSC}$ in $\mathrm{HCCs}$, as reflected by the protection of Foxd3 from MDM2-induced polyubiquitination and degradation when Foxd3 was constitutively acetylated in our experiments. Knockdown of Foxd3 promoted the entry of
$\mathrm{CD}^{+}{ }^{+} \mathrm{CSC}$ into cell cycle, and decreased their self-renewal. As a consequence, Foxd3-deficient populations lost features characteristic of CSCs, i.e., quiescence and self-renewal, and were susceptible to chemotherapy. In this sense, Tyr metabolism not only meets the energy requirement of $\mathrm{CD} 13^{+} \mathrm{CSCs}$, but also is essential for the maintenance of this subpopulation. Targeting the metabolic "Achilles heel" of CD13+ CSCs by nitisinone in parallel to tumor debulking by chemotherapy may be required for optimal therapeutic specificity yielding the most durable tumor remission in HCCs. Last but not least, nitisinone is an imperfect treatment, in as much as it markedly increases circulating Tyr. Managing high Tyr by using a lower protein diet to minimize adverse effects including skin rash and corneal keratopathy will be required [30].

\section{Electronic Supplementary Material}

Supplementary materials are available at Cancer Research and Treatment website (https://www.e-crt.org).

\section{Conflicts of Interest}

Conflict of interest relevant to this article was not reported.

\section{Acknowledgments}

This work was supported by Funds of the Shanghai Board of Health (No. 184080). We thank members of our laboratory for helpful discussions.

\section{References}

1. Bray F, Ferlay J, Soerjomataram I, Siegel RL, Torre LA, Jemal A. Global cancer statistics 2018: GLOBOCAN estimates of incidence and mortality worldwide for 36 cancers in 185 countries. CA Cancer J Clin. 2018;68:394-424.

2. Bruix J, Sherman M; American Association for the Study of Liver Diseases. Management of hepatocellular carcinoma: an update. Hepatology. 2011;53:1020-2.

3. European Association for the Study of the Liver. EASL clinical practice guidelines: management of alcohol-related liver disease. J Hepatol. 2018;69:154-81.

4. Phi LT, Sari IN, Yang YG, Lee SH, Jun N, Kim KS, et al. Cancer stem cells (CSCs) in drug resistance and their therapeutic implications in cancer treatment. Stem Cells Int. 2018;2018:5416923.

5. Yamashita T, Wang XW. Cancer stem cells in the development of liver cancer. J Clin Invest. 2013;123:1911-8.

6. Yang ZF, Ngai P, Ho DW, Yu WC, Ng MN, Lau CK, et al.
Identification of local and circulating cancer stem cells in human liver cancer. Hepatology. 2008;47:919-28.

7. Ma S, Chan KW, Hu L, Lee TK, Wo JY, Ng IO, et al. Identification and characterization of tumorigenic liver cancer stem/ progenitor cells. Gastroenterology. 2007;132:2542-56.

8. Haraguchi N, Ishii H, Mimori K, Tanaka F, Ohkuma M, Kim $\mathrm{HM}$, et al. CD13 is a therapeutic target in human liver cancer stem cells. J Clin Invest. 2010;120:3326-39.

9. Christ B, Stock P, Dollinger MM. CD13: waving the flag for a novel cancer stem cell target. Hepatology. 2011;53:1388-90.

10. Luan $Y, X u$ W. The structure and main functions of aminopeptidase N. Curr Med Chem. 2007;14:639-47.

11. Sancho P, Barneda D, Heeschen C. Hallmarks of cancer stem cell metabolism. Br J Cancer. 2016;114:1305-12.

12. Deshmukh A, Deshpande K, Arfuso F, Newsholme P, Dharmarajan A. Cancer stem cell metabolism: a potential target for cancer therapy. Mol Cancer. 2016;15:69. 
13. Cairns RA, Harris IS, Mak TW. Regulation of cancer cell metabolism. Nat Rev Cancer. 2011;11:85-95.

14. Li L, Bhatia R. Stem cell quiescence. Clin Cancer Res. 2011;17: 4936-41.

15. Drost J, Clevers H. Organoids in cancer research. Nat Rev Cancer. 2018;18:407-18.

16. Mao P, Joshi K, Li J, Kim SH, Li P, Santana-Santos L, et al. Mesenchymal glioma stem cells are maintained by activated glycolytic metabolism involving aldehyde dehydrogenase $1 \mathrm{~A} 3$. Proc Natl Acad Sci U S A. 2013;110:8644-9.

17. Ferreira LM, Hebrant A, Dumont JE. Metabolic reprogramming of the tumor. Oncogene. 2012;31:3999-4011.

18. Lock E, Ranganath LR, Timmis O. The role of nitisinone in tyrosine pathway disorders. Curr Rheumatol Rep. 2014;16:457.

19. Ait-Si-Ali S, Carlisi D, Ramirez S, Upegui-Gonzalez LC, Duquet A, Robin P, et al. Phosphorylation by p44 MAP Kinase/ERK1 stimulates CBP histone acetyl transferase activity in vitro. Biochem Biophys Res Commun. 1999;262:157-62.

20. Santos AN, Langner J, Herrmann M, Riemann D. Aminopeptidase N/CD13 is directly linked to signal transduction pathways in monocytes. Cell Immunol. 2000;201:22-32.

21. Yang JY, Zong CS, Xia W, Yamaguchi H, Ding Q, Xie X, et al. ERK promotes tumorigenesis by inhibiting FOXO3a via MDM2-mediated degradation. Nat Cell Biol. 2008;10:138-48.

22. Pauklin S, Vallier L. The cell-cycle state of stem cells determines cell fate propensity. Cell. 2013;155:135-47.

23. Merlos-Suarez A, Barriga FM, Jung P, Iglesias M, Cespedes MV, Rossell D, et al. The intestinal stem cell signature identi- fies colorectal cancer stem cells and predicts disease relapse. Cell Stem Cell. 2011;8:511-24.

24. Ito K, Suda T. Metabolic requirements for the maintenance of self-renewing stem cells. Nat Rev Mol Cell Biol. 2014;15:24356.

25. Possemato R, Marks KM, Shaul YD, Pacold ME, Kim D, Birsoy $\mathrm{K}$, et al. Functional genomics reveal that the serine synthesis pathway is essential in breast cancer. Nature. 2011;476:346-50.

26. Wensveen FM, Alves NL, Derks IA, Reedquist KA, Eldering E. Apoptosis induced by overall metabolic stress converges on the Bcl-2 family proteins Noxa and Mcl-1. Apoptosis. 2011;16: 708-21.

27. Kim HM, Haraguchi N, Ishii H, Ohkuma M, Okano M, Mimori K, et al. Increased CD13 expression reduces reactive oxygen species, promoting survival of liver cancer stem cells via an epithelial-mesenchymal transition-like phenomenon. Ann Surg Oncol. 2012;19 Suppl 3:S539-48.

28. Wellen KE, Thompson CB. A two-way street: reciprocal regulation of metabolism and signalling. Nat Rev Mol Cell Biol. 2012;13:270-6.

29. Yong JS, Intriago-Baldeon DP, Lam EW. FOXD3 controls pluripotency through modulating enhancer activity. Stem Cell Investig. 2016;3:17.

30. Stewart RM, Briggs MC, Jarvis JC, Gallagher JA, Ranganath L. Reversible keratopathy due to hypertyrosinaemia following intermittent low-dose nitisinone in alkaptonuria: a case report. JIMD Rep. 2014;17:1-6. 